\title{
Égalité entre les sexes (et autres identités), diversité et inclusion en chirurgie
}

Les opinions exprimées dans cet éditorial sont celles de l'auteur et ne représentent pas nécessairement celles de l'Association médicale canadienne ou ses filiales.

e retard qu'accuse la chirurgie par rapport à bien d'autres domaines de la médecine dans des sujets comme la diversité, l'inclusion et l'égalité entre les sexes demeure une préoccupation majeure. Loin d'être un problème particulier au Canada ou en Amérique du Nord, le phénomène est mondial. Mais avant même qu'il soit possible de discuter d'égalité en chirurgie, il s'avère que des préjugés tenaces sont souvent en cause, certains étant à l'évidence involontaires, alors que d'autres sont potentiellement intentionnels. À titre d'exemple, des écoles de médecine au Japon ont été accusées ces dernières années d'avoir truqué leur processus d'admission en attribuant de façon factice aux femmes qui présentaient des demandes des notes inférieures à celles des candidats masculins ${ }^{1}$. Cet acte de mauvaise foi a eu pour conséquence de faire stagner la proportion d'étudiantes en médecine à $30 \%$. Au Canada, le nombre de femmes en médecine a finalement dépassé la parité hommes-femmes, car dans bon nombre de facultés, les femmes sont en fait plus nombreuses que les hommes. Fait intéressant toutefois, cette réalité ne s'applique pas à tous champs de la chirurgie. Selon la Fédération des médecins spécialistes du Québec, en 2019, 45 \% des médecins spécialistes au Québec sont des femmes, alors que seulement $10 \%$ des chirurgiens cardiovasculaires et thoraciques et $19 \%$ des chirurgiens orthopédiques sont des femmes. Certes, il existe d'importantes différences culturelles et socioéconomiques dans le monde de même que des rites enracinés qui véhiculent des préjugés implicites basés sur le genre dans chaque système. Vu sous cet angle, il n'est pas étonnant que des problèmes exprimés de façon implicite ou explicite se fassent sentir dans les champs de la chirurgie.

Ainsi qu'il est fait mention dans le livre blanc publié par l'American Surgical Association sur l'égalité, la diversité et l'inclusion, cette question doit être abordée à tous les niveaux ${ }^{2}$. Le recrutement, le financement, l'allocation de ressources et les subventions sont tous des aspects susceptibles d'améliorer la situation. Le biais de sélection des sujets dans les études doit aussi faire l'objet d'une amélioration ${ }^{3,4}$. De toute évidence, les efforts en faveur de la diversité en général amélioreront aussi la productivité, la collégialité et les soins aux patients.
Nous devons aussi mesurer nos progrès, nous efforcer de poursuivre notre travail de sensibilisation et toujours viser l'amélioration. Nous avons tout mis en œuvre pour jouer un rôle actif en rendant compte de ces progrès dans le Fournal canadien de chirurgie. Plus précisément, $50 \%$ des articles soumis portant sur les préjugés sexistes en chirurgie ont été publiés. Nous avons également favorisé la tenue de débats animés et accueilli les commentaires dans notre section Discussions en chirurgie. Dorénavant, la question sera de savoir comment nous pouvons nous surpasser. Au journal, nous tentons d'analyser des méthodes qui pourraient mieux combler le manque de connaissances sur l'égalité entre les sexes et l'équité raciale et répondre aux autres questions d'inclusion afin d'assurer dans l'ensemble une recherche de haute qualité et fondée sur une solide méthodologie. On tiendra compte des suggestions de sujets de discussion en ligne lesquels serviront de plateforme à la diffusion hâtive d'idées et de résultats, et ce, même avant la révision par des pairs. La création d'un forum avec des sujets présentant un intérêt particulier constitue une autre possibilité. Nous aimerions solliciter des commentaires de nos lecteurs, quels que soient leur sexe, leur race et leur groupe en ce qui concerne les éventuels mécanismes qui mènent à la poursuite de ces efforts.

\section{Edward J. Harvey, MD; Chad G. Ball, MD \\ Corédacteurs en chef, fournal canadien de chirurgie}

Intérêts concurrents : E.J. Harvey est médecin chef chez Greybox Solutions; il est cofondateur et directeur de l'innovation médicale de NXTSens Inc, cofondateur et médecin chef de MY01 Inc. et cofondateur et directeur de Strathera Inc. Il bénéficie du soutien institutionnel de J \& J, DePuy Synthes, Stryker et Zimmer, et il fait partie du conseil d'administration de l'Orthopedic Trauma Association et de l'Association canadienne d'orthopédie. Aucun déclaré par C.G. Ball.

DOI: $10.1503 /$ cjs.015019

\section{Références}

1. Ramzy A, Ueno H. Japanese medical school accused of rigging admissions to keep women out. New York Times. Le 3 août 2018.

2. West MA, Hwang S, Maier RV, et coll. Ensuring equity, diversity and inclusion in academic surgery: an American Surgical Association white paper. Ann Surg. 2018; 268:403-7.

3. Lillemoe KD. Joint statement by the Surgery Journal Editors Group 2018. Ann Surg. 2018; 267:991.

4. Mansukhani NA, Yoon DY, Teter KA, et coll. Determining if sex bias exists in human surgical clinical research. FAMA Surg 2016;151:1022-30. 